\title{
Extramural Perineural Invasion in pT3 and pT4 Gastric Carcinomas
}

\author{
Alejandro España-Ferrufino \\ Leonardo S. Lino-Silva \\ Rosa A. Salcedo-Hernández ${ }^{1}$ \\ Departments of Gastrointestinal Pathology and \\ ${ }^{1}$ Surgical Oncology, Instituto Nacional de \\ Cancerología (INCan), Mexico City, Mexico \\ Received: August 16, 2017 \\ Revised: October 20, 2017 \\ Accepted: November 1, 2017 \\ Corresponding Author \\ Leonardo S. Lino-Silva, MD \\ Department of Gastrointestinal Pathology, \\ Instituto Nacional de Cancerología, Av. San \\ Fernando 22 Col. Sección XVI, CP. 14080, México \\ City, México \\ Tel: +52-55-5628-0400 (ext. 11068) \\ Fax: $+52-55-56280401$ \\ E-mail: saul.lino.sil@gmail.com
}

Background: Perineural invasion (PNI) is widely studied in malignant tumors, and its prognostic significance is well demonstrated. Most studies have focused on evaluating the mural PNI (mPNI); however, extramural PNI (ePNI) may influence the prognosis in gastric cancer. We evaluated the prognostic value of ePNI compared with $\mathrm{mPNI}$ in gastric cancer in this observational comparative cross-sectional study. Methods: Seventy-three pT3 and pT4 gastric carcinomas with PNI were evaluated. Forty-eight $(65.7 \%)$ were in the $\mathrm{mPNI}$ group and the remaining in the ePNI group. Results: Clinicopathologic characteristics between the two groups were similar, except for the outcomes. The 5-year disease-specific survival (DSS) rate was 64\% for the mPNI group and $50 \%$ for the ePNI group $(p=.039)$, a difference that did not remain significant in multivariate analysis. The only independent adverse prognostic factor in multivariate analysis was the presence of lymph node metastasis (hazard ratio, $1.757 ; 95 \%$ confidence interval, 1.082 to $2.854 ; p=.023$ ). Conclusions: We demonstrated the prognostic effect of ePNI for DSS in surgically resected pT3pT4 gastric cancer patients. ePNI could be considered in the staging and prognostic systems of gastric cancer to stratify patients with a high risk of recurrence.

Key Words: Stomach neoplasms; Perineural invasion; Survival; Prognostic factor; Neoplasms
Gastric cancer is the fifth most common cancer worldwide, with about one million new cases reported in 2012 (6.8\% of the total). ${ }^{1}$ About $70 \%$ of cases occur in developed and developing countries a half of them are presented in East Asia (mainly in China) and twice as often in men over women. Although the incidence of gastric cancer has declined in recent decades, it remains the third leading cancer-related cause of death worldwide with 723,000 , deaths representing $8.8 \%$ of all cancer deaths. ${ }^{1}$ The prognosis in advanced stages of gastric cancer is poor, even with the use of chemotherapy or other adjuvant treatments.

Perineural invasion (PNI) is one of the pathological factors widely studied in malignant neoplasms with a well-established prognostic significance in head and neck neoplasia and prostate cancer. ${ }^{2} \mathrm{PNI}$ is related to a more aggressive behavior of the neoplasia and poor prognosis in several malignancies. ${ }^{3}$ However, there is no universal definition of PNI. Several pathologists simply define PNI as the presence of neoplastic cells in, around or through the nerves, while others require the presence of tumor cells within any of the epineurium, perineurium, or endoneurium. The most accepted one was defined by Batsakis, ${ }^{4}$ defining it as the invasion of tumor cells in, around, and through nerves. Other authors define PNI according to the location of the neoplastic cells with regards to the layers of the nerve sheath (outer epineurium, perineurium, and inner endoneurium). Liebig et al. ${ }^{5}$ defined PNI as the presence of neoplastic cells in any three layers of the nerve sheath or in foci outside the nerve sheath with the involvement of $33 \%$ of the nerve circumference. The criterion of PNI defined as cancer cells inside the perineurium and surrounding at least $33 \%$ of the nerve circumference showed a perfect interobserver concordance.

PNI was once thought to be an extension of lymphatic metastasis, but recent studies have shown that lymphatic channels do not penetrate the layers of the nerve sheath. ${ }^{6-8}$ Studies on prostate and pancreatic cancer showed that in PNI there is an interaction of reciprocal neurotrophic factors between the neoplastic cells and the nerves, like nerve growth factor (NGF), brain-derived neurotrophic factor, neurotrophin 3, and neurotrophin., ${ }^{9,10}$ Okada et al. ${ }^{11}$ reported that exogenous NGF leads to a dose-dependent increase in matrix metalloproteinase 2 expression and invasion in neoplastic cells of the pancreas.

In a systematic review and meta-analysis of 30,590 cases on PNI in gastric cancer, Deng et al..$^{12}$ found PNI as an independent predictor of recurrence, as well as to affecting disease-free survival and overall survival in patients with gastric cancer undergoing 
curative resection. Although most authors focus their attention on evaluating mural perineural invasion (mPNI, invasion of the nerve plexus in the proper muscle and submucosal muscle), there are studies in gastrointestinal cancers (especially colon cancer) that demonstrate that the PNI outside the muscular wall (extramural PNI [ePNI]) has a worse prognosis. ${ }^{13}$ To date, there are no studies subclassifying PNI into mPNI and ePNI in gastric carcinoma.

Our goal was to confirm whether the prognosis associated with PNI is determined by ePNI rather than mPNI under the hypothesis that the patients with ePNI will show worse diseasespecific survival (DSS) than those with mPNI, in pT3-pT4 carcinomas in clinical stages II and III.

\section{MATERIALS AND METHODS}

From the database of patients with gastric adenocarcinomas from 2005 to 2015 at the national referral cancer center in Mexico, we searched for patients who underwent curative resection of gastric adenocarcinoma in clinical stages II and III, with pathological tumor stages pT3 and pT4 and with PNI described in the pathological report. Most patients at our institution were at stage IV disease at presentation or in poor clinical conditions and they did not receive surgery. In our center, the same surgical team performs around 30 gastric surgeries per year. Gastrectomy with D2 lymphadenectomy was the standard surgical procedure in all cases. The standard adjuvant therapy was capecitabine/ oxaliplatin in selected patients (especially in those who had pathological risk factors and/or low lymph node counts). Data on patient demographics, tumor localization, operation, and histopathological study were recorded. The staging was determined by clinical, radiological, and histopathological data according to the American Joint Commission on Cancer pTNM system (7th edition, 2010). ${ }^{14}$

For the selected cases ( $\mathrm{n}=73$ ), two pathologists with expertise in gastrointestinal pathology separately evaluated a median of five hematoxylin and eosin stained slides (range, 3 to 8) for evaluation of PNI and other pathologic features. Each pathologist was blind to the patient data and the diagnosis of the other pathologist. The criterion of PNI was cancer cells inside the perineuroum involving at least $33 \%$ of the nerve circumference (Fig. 1). ${ }^{5,15}$ When PNI was present in the submucosa or muscularis propria, the pathologist classified the case as mPNI. If the the tumor invaded a nerve located beyond the muscularis propria (subserosal tissue or adventitia), it was classified as ePNI. When the muscular layer could not be identified due to tumor destruction, an imaginary line was drawn between the breakpoints of

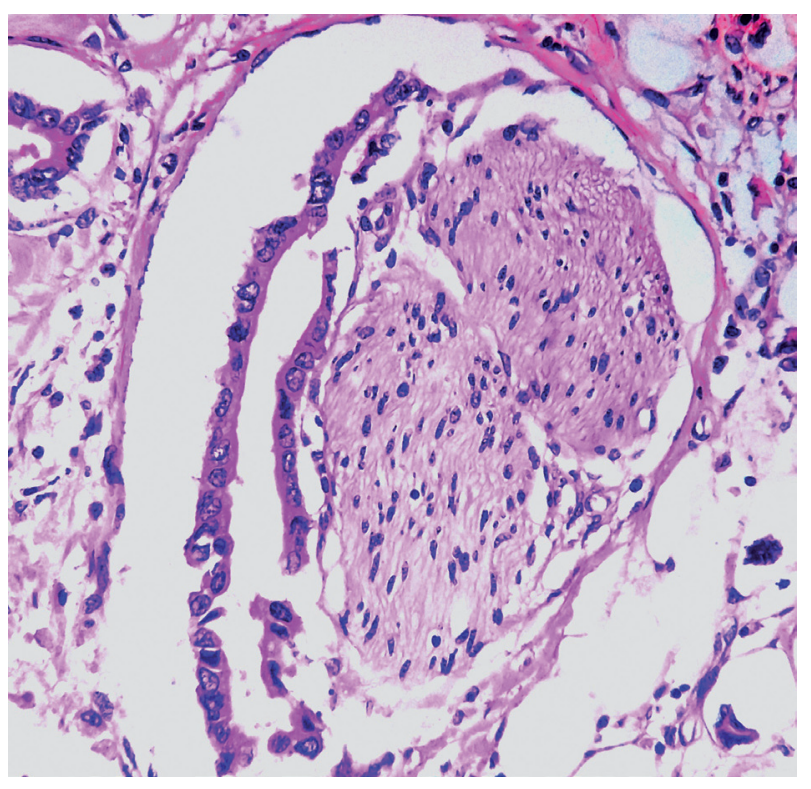

Fig. 1. Histologic microphotography showing perineural space invasion. Note that neoplastic glands invade perineurium and encases at least $33 \%$ of the circumference of the nerve.

intact muscularis propria. Discordant cases were reviewed by both pathologists under the same microscope for consensus. The tumor was classified as intestinal adenocarcinoma when glandular differentiation was clearly demonstrated and as diffuse adenocarcinoma when there was no glandular differentiation and the tumor was composed of individual cells with or without signet ring cells.

\section{Statistic analysis}

Data were analyzed using the Statistical Package for Social Sciences ver.12.0 (SPSS Inc., Chicago, IL, USA). Kappa statistic for interobserver concordance regarding PNI was performed before consensus between the pathologists. A comparison of the means was performed with an unpaired Student $t$ test. Chisquare and Fisher exact tests were performed to examine associations between categorical variables. In all cases, p-values were two-sided, and a statistical significance was accepted when $\mathrm{p}<.05$.

\section{Survival analysis}

The primary end-point was DSS defined as cancer death, determined from the date of the first treatment, including palliative care (event) or last follow-up (censored). The DSS curves were estimated using the Kaplan-Meier method. The univariate Cox regression model was used to examine the association of variables with DSS. Significant characteristics in the univariate analysis (variables with a $\mathrm{p}<.05$ ) were introduced into a multi- 
variate model of Cox proportional hazards in addition to age and sex.

This study was approved by the Institutional Review Board and Ethics Committe of the National Cancer Institute of Mexico with a waiver of informed consent because of the retrospective nature of the study (IRB No. CEI.16/117).

Table 1. Clinicopathologic data comparison between 73 gastric carcinomas in pT3 and pT4 stage according perineural invasion

\begin{tabular}{|c|c|c|c|}
\hline Variable & Intramural $(n=25)$ & Extramural $(n=48)$ & $p$-value ${ }^{a}$ \\
\hline Median age (Q1-Q3 range) & $56(46-65)$ & $58(47-65)$ & .783 \\
\hline \multicolumn{4}{|l|}{ Sex } \\
\hline Female & $12(48)$ & $23(47.9)$ & .617 \\
\hline Male & $13(52)$ & $25(52.1)$ & \\
\hline \multicolumn{4}{|l|}{ pT } \\
\hline pT3 & $15(60)$ & $21(43.8)$ & .247 \\
\hline pT4a & $10(40)$ & $24(50)$ & \\
\hline $\mathrm{pT} 4 \mathrm{~b}$ & 0 & $3(6.3)$ & \\
\hline \multicolumn{4}{|l|}{ Nodal metastasis } \\
\hline NO & $5(20)$ & $8(16.7)$ & .423 \\
\hline N1 & $12(48)$ & $25(52.1)$ & \\
\hline N2 & $8(32)$ & 14 (29.2) & \\
\hline N3 & 0 & $1(2.1)$ & \\
\hline \multicolumn{4}{|l|}{ Location } \\
\hline Proximal third & $5(20)$ & $11(22.9)$ & .759 \\
\hline Medim third & $7(28)$ & $12(25)$ & \\
\hline Distal third & $13(52)$ & $25(52.1)$ & \\
\hline Median number of dissected lymph nodes (Q1-Q3 range) & $26(19-36)$ & $26(19-39)$ & .818 \\
\hline Median number of positive lymph nodes (Q1-Q3 range) & $7(2-11)$ & $10(3-22)$ & .933 \\
\hline \multicolumn{4}{|l|}{ Distant metastases } \\
\hline No & $18(72)$ & $33(68.8)$ & .774 \\
\hline Yes & $7(28)$ & $15(31.2)$ & \\
\hline \multicolumn{4}{|l|}{ Lymphovascular invasion } \\
\hline No & $4(16)$ & 7 (14.6) & .872 \\
\hline Yes & $21(84)$ & $41(85.4)$ & \\
\hline \multicolumn{4}{|l|}{ Grade } \\
\hline Well differentiated & $2(8)$ & $4(8.3)$ & .405 \\
\hline Moderately differentiated & $19(76)$ & $41(85.4)$ & \\
\hline Poorly differentiated & $4(16)$ & $3(6.3)$ & \\
\hline \multicolumn{4}{|l|}{ Clinical stage } \\
\hline Stage II & $7(28)$ & $10(20.8)$ & .770 \\
\hline Stage III & $11(44)$ & $22(45.8)$ & \\
\hline Stage IV & $7(28)$ & $16(33.4)$ & \\
\hline \multicolumn{4}{|l|}{ Resection } \\
\hline Ro & $24(96)$ & $44(91.7)$ & .487 \\
\hline R1 & $1(4)$ & $4(8.3)$ & \\
\hline \multicolumn{4}{|l|}{ Adjuvant treatment } \\
\hline No & $3(12)$ & $16(33.3)$ & .137 \\
\hline Yes & $22(88)$ & $32(66.7)$ & \\
\hline \multicolumn{4}{|l|}{ Overall recurrence } \\
\hline No & $12(48)$ & $18(37.5)$ & .231 \\
\hline Yes & $13(52)$ & $30(62.5)$ & \\
\hline \multicolumn{4}{|l|}{ Outcome } \\
\hline Alive free of disease & $9(36)$ & $5(10.4)$ & .023 \\
\hline Dead with disease & $6(24)$ & $14(29.2)$ & \\
\hline Alive with disease & $10(40)$ & $22(45.8)$ & \\
\hline Dead without disease & 0 & 7 (14.6) & \\
\hline Median follow-up (Q1-Q3 range, mo) & $13(8-51)$ & $11(5-19)$ & .052 \\
\hline 5-Year disease-specific survival (\%) & 64 & 50 & .039 \\
\hline
\end{tabular}

Values are presented as median (range) or number (\%).

${ }^{a}$ Chi square test or Kruskal-Wallis test. 


\section{RESULTS}

\section{Clinicopathologic characteristics}

Features of the patient cohort $(n=73)$ are summarized in Table 1 . The median age was 56 years old, ranging from 46 to 65 years. Thirty-eight patients (52\%) were male and $35(48 \%)$ were female. In total, 68 patients (93\%) received total gastrectomy and five received subtotal gastrectomy. Surgery was performed by laparoscopy in 15 cases (20.5\%). During the laparotomy or laparoscopy, 22 patients (30.1\%) presented with resectable peritoneal, hepatic or splenic metastasis, and all of them were fully resected. Major complications occurred in nine patients (12.3\%; i.e., anastomotic leakage, septicemia, dehiscence). PNI was verified in all cases, with a majority $(65.7 \%)$ in the ePNI group, and the kappa statistic was 1.0 for concordance between observers. There was no difference in clinicopathologic features between the groups including $\mathrm{pT}$ and $\mathrm{pN}$ stage (Table 1). Patients with ePNI were less likely to be free of disease ( $10.4 \%$ vs $36 \%$ ). The percentage of each histologic type of the tumor was also similar between the groups.

\section{Outcome}

The median follow-up time was $12 \pm 24.6$ months, ranging from 5 to 116 months. In this timeframe, 43 patients had recurrence (48.9\%): 33 distant and 10 local. The median time to recurrence was 5 months, with a range from 3 to 20 months. The incidence of recurrence was similar between the groups. Of the patient cohort, $19.2 \%$ of all patients were alive and free of disease, $27.4 \%$ died from the disease, $43.8 \%$ were alive with disease, and $9.6 \%$ died from causes not related to gastric carcinoma. Patients with ePNI had a higher mortality rate than patients with $\mathrm{mPNI}$ $(29.2 \%$ vs $24 \%)(\mathrm{p}=.023)$.

\section{Survival analysis}

The 5 -year DSS was $60 \%$. Univariate analysis is summarized in Table 2. Lymph node metastasis, tumor stage pT4b, presence of ePNI and recurrence were predictors of increased mortality. Patients with PNI showed differences in survival between the groups, with 5-year DSS of $64 \%$ for 25 patients with mPNI compared with $50 \%$ for 48 patients in the ePNI group ( $\mathrm{p}=.039)$ (Fig. 2). Among the patients in the ePNI group, 14 were dead of disease with a median of 16 months, and 22 patients were alive with disease (recurrence). The median DSS of the patients in the ePNI group was 16 months and for the patients in the mPNI group was 46 months. Most patients (74\%) received adjuvant therapy with heterogeneous modalities for subanalysis. All patients
Table 2. Univariate analysis of 73 gastric carcinomas ${ }^{a}$

\begin{tabular}{|c|c|c|}
\hline Variable & $\begin{array}{l}\text { 5-Year disease- } \\
\text { specific survival (\%) }\end{array}$ & $p$-value \\
\hline \multicolumn{3}{|l|}{ Sex } \\
\hline Male & 49 & .591 \\
\hline Female & 65 & \\
\hline \multicolumn{3}{|l|}{ Pathologic tumoral category } \\
\hline pT3 & 67 & .023 \\
\hline pT4a & 43 & \\
\hline pT4b & 0 & \\
\hline \multicolumn{3}{|l|}{ Clinical stage } \\
\hline$\|$ & 85 & \\
\hline III & 36 & .116 \\
\hline IV & 27 & \\
\hline \multicolumn{3}{|l|}{ Lymph node metastasis } \\
\hline No & 90 & .001 \\
\hline Yes & 42 & \\
\hline \multicolumn{3}{|l|}{ Distant metastases } \\
\hline No & 56 & .880 \\
\hline Yes & 46 & \\
\hline \multicolumn{3}{|l|}{ Lymphovascular invasion } \\
\hline No & 90 & .290 \\
\hline Yes & 50 & \\
\hline \multicolumn{3}{|l|}{ Perineural invasion } \\
\hline Intramural & 64 & .039 \\
\hline Extramural & 49 & \\
\hline \multicolumn{3}{|l|}{ Grade } \\
\hline Well differentiated & 50 & .619 \\
\hline Moderately differentiated & 56 & \\
\hline Poorly differentiated & 50 & \\
\hline \multicolumn{3}{|l|}{ Adjuvant therapy } \\
\hline No & 62 & .713 \\
\hline Yes & 66 & \\
\hline \multicolumn{3}{|l|}{ Resection } \\
\hline Ro & 52 & .783 \\
\hline $\mathrm{R} 1$ & 60 & \\
\hline \multicolumn{3}{|l|}{ Recurrence } \\
\hline No & 87 & .007 \\
\hline Yes & 42 & \\
\hline
\end{tabular}

with nodal metastasis, lymphovascular invasion and R1 resections received adjuvant therapy. R1 resections had a higher 5-year survival than $\mathrm{R} 0$ resections, which can be explained by the rate of adjuvant therapy in the R1 group; however, the difference was not statistically significant.

The only independent adverse prognostic factor identified in multivariate analysis was lymph node metastasis (hazard ratio, $1.757 ; 95 \%$ confidence interval, 1.082 to $2.854 ; \mathrm{p}=.023$ ). The remaining factors including ePNI were not associated with poor DSS as independent factors (Table 3). 


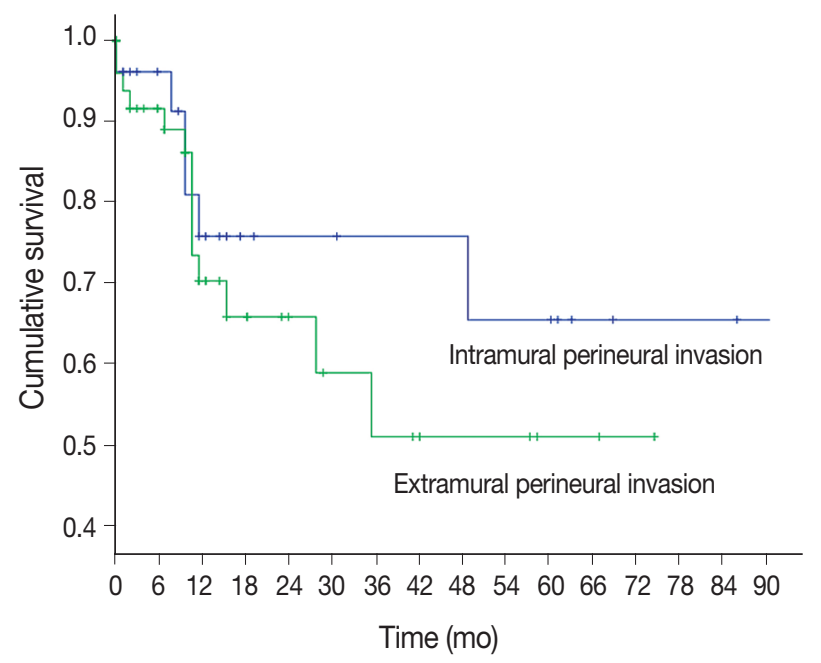

Fig. 2. Five-year disease-specific survival of 73 patients with gastric carcinoma divided by perineural invasion.

Table 3. Multivariate analysis of 73 gastric carcinomas

\begin{tabular}{lcccc}
\hline Variable & $\begin{array}{c}\text { Cox } \\
\text { hazard } \\
\text { ratio }\end{array}$ & $95 \% \mathrm{Cl}$ & $\begin{array}{c}\mathrm{p}- \\
\text { value }\end{array}$ \\
\hline Lymph node metastasis (yes vs no) & 1.757 & $1.082-2.854$ & .023 \\
Recurrence (yes vs no) & 1.594 & $0949-2.676$ & .078 \\
Pathologic T category (pT3 vs pT4). & 1.187 & $0.453-3.110$ & .727 \\
Extramural perineural invasion (yes vs no). & 1.096 & $0.393-3.054$ & .861 \\
\hline
\end{tabular}

$\mathrm{Cl}$, confidence interval.

\section{DISCUSSION}

PNI has been identified in gastric cancer with a median of $40.9 \%(6.8 \%-75.6 \%){ }^{12}$ This is the first study subclassifying $\mathrm{PNI}$ into $\mathrm{mPNi}$ and ePNI in gastric cancer. We found ePNI to be present in equal sex distribution, with a median age of 58 years, more frequent in pT4a tumors, in patients with lymphovascular invasion and associated with a lower DSS compared with mPNI.

In gastric cancer, the prognostic significance of PNI is clear. Tanaka et al. ${ }^{16}$ reported that PNI is a determinant in the prognosis of advanced cancer and found that $80 \%$ of patients with peritoneal recurrence had PNI whereas Duraker et al. ${ }^{17}$ showed that PNI was present in $59.9 \%$ of patients with disease progression. Bilici et al. ${ }^{18}$ found that the mean survival of patients with PNI was shorter than patients without PNI (60.3 months vs 27.9 months, respectively). Tianhang et al..$^{19}$ found a significant relationship between clinical stage and PNI; both were independent prognostic factors. Finally, Selcukbiricik et al ${ }^{20}$ and Jiang et $a l .{ }^{21}$ demosntrated that PNI is an independent prognostic factor associated with tumor size $(>5 \mathrm{~cm})$, lymphovascular inva- sion, pT4 tumors, lymph node metastasis, and advanced stage. ${ }^{21}$

However, we think that this observations are due to ePNI rather than mPNI based on findings like those by Ueno et al. ${ }^{13}$ who analyzed 364 patients with rectal cancer, dividing PNI into PNI-0 (absence of IPN), PN-1 (less than five PNI foci in the first $10 \mathrm{~mm}$ measured from the outer muscle layer), PN-2 (five or more foci or $10 \mathrm{~mm}$ or more from the muscularis propria), and reported a 5 -year survival of $74 \%, 50 \%$, and $22 \%$, respectively. They showed that the degrees of PNI were independently associated with local recurrence and long-term survival regardless of tumor depth and lymph node metastasis. ${ }^{13}$ We applied a similar approach to gastric cancer, demonstrating similar results; an independent worse DSS of patients with ePNI compared with those with mPNI.

The perineural space is a potential route for tumor spread in gastric adenocarcinoma, especially outside muscularis propria in patients with $\mathrm{pT} 3$ and $\mathrm{pT} 4$ tumors. While in multivariate analysis their association could not be demonstrated as an independent factor for survival, it is clear that it is associated with lower median survival and 5-year DSS. We believe that this difference does not have significance in multivariate analysis in part because of the poor prognosis of these patients due to the advanced pathological stage and possibly inaccurate lymph node stage (some cases in our series had less than 25 lymph nodes in "D2" gastrectomy). However, in order to decrease these potential biases, we only used cases in $\mathrm{pT} 3$ and $\mathrm{pT} 4$ stages because these are the tumors that invade beyond muscularis propria.

It is plausible that ePNI could be considered in the staging and prognostic systems in gastric cancer to stratify patients with a high risk of recurrence. Our results need to be confirmed in a larger series; however, this study provide us with information on the possibility that PNI behaves differently depending on how it is evaluated, which can explain the lack of consensus on the true prognostic value of PNI.

\section{Conflicts of Interest}

No potential conflict of interest relevant to this article was reported.

\section{REFERENCES}

1. Ferlay J, Soerjomataram I, Ervik M, et al. GLOBOCAN 2012 v1.0, cancer incidence and mortality worldwide: IARC CancerBase No. 11. IARC CancerBase No. 11 [Internet]. Lyon: International Agency for Research on Cancer, 2013 [cited 2017 Dec 10]. Available from: 
http://globocan.iarc.fr.

2. Fagan JJ, Collins B, Barnes L, D’Amico F, Myers EN, Johnson JT. Perineural invasion in squamous cell carcinoma of the head and neck. Arch Otolaryngol Head Neck Surg 1998; 124: 637-40.

3. Liebig C, Ayala G, Wilks JA, Berger DH, Albo D. Perineural invasion in cancer: a review of the literature. Cancer 2009; 115: 3379-91.

4. Batsakis JG. Nerves and neurotropic carcinomas. Ann Otol Rhinol Laryngol 1985; 94(4 Pt 1): 426-7.

5. Liebig C, Ayala G, Wilks J, et al. Perineural invasion is an independent predictor of outcome in colorectal cancer. J Clin Oncol 2009; 27: 5131-7.

6. Hassan MO, Maksem J. The prostatic perineural space and its relation to tumor spread: an ultrastructural study. Am J Surg Pathol 1980; 4: 143-8.

7. Reina MA, López A, Villanueva MC, de Andrés JA, León GI. Morphology of peripheral nerves, their sheaths, and their vascularization. Rev Esp Anestesiol Reanim 2000; 47: 464-75.

8. Ayala GE, Wheeler TM, Shine HD, et al. In vitro dorsal root ganglia and human prostate cell line interaction: redefining perineural invasion in prostate cancer. Prosate 2001; 49: 213-23.

9. Boyd JG, Gordon T. Neurotrophic factors and their receptors in axonal regeneration and functional recovery after peripheral nerve injury. Mol Neurobiol 2003; 27: 277-324.

10. Ketterer K, Rao S, Friess H, Weiss J, Büchler MW, Korc M. Reverse transcription-PCR analysis of laser-captured cells points to potential paracrine and autocrine actions of neurotrophins in pancreatic cancer. Clin Cancer Res 2003; 9: 5127-36.

11. Okada Y, Eibl G, Guha S, Duffy JP, Reber HA, Hines OJ. Nerve growth factor stimulates MMP-2 expression and activity and increases invasion by human pancreatic cancer cells. Clin Exp Metastasis 2004; 21: 285-92.
12. Deng J, You Q, Gao Y, et al. Prognostic value of perineural invasion in gastric cancer: a systematic review and meta-analysis. PLoS One 2014; 9: e88907.

13. Ueno $\mathrm{H}$, Hase K, Mochizuki H. Criteria for extramural perineural invasion as a prognostic factor in rectal cancer. Br J Surg 2001; 88: 994-1000.

14. Edge SB, Byrd DR, Compton CC, Fritz AG, Greene FL, Trotti A. American Joint Committee on Cancer Cancer staging manual. New York: Springer, 2010.

15. Ceyhan GO, Liebl F, Maak M, et al. The severity of neural invasion is a crucial prognostic factor in rectal cancer independent of neoadjuvant radiochemotherapy. Ann Surg 2010; 252: 797-804.

16. Tanaka A. Watabe T, Okuno K, Yasutomi M. Perineural invasion as a predictor of recurrence of gastric cancer. Cancer 1994; 73: 550-5.

17. Duraker N, Sişman S, Can G. The significance of perineural invasion as a prognostic factor in patients with gastric carcinoma. Surg Today 2003; 33: 95-100.

18. Bilici A, Seker M, Ustaalioglu BB, et al. Prognostic significance of perineural invasion in patients with gastric cancer who underwent curative resection. Ann Surg Oncol 2010; 17: 2037-44.

19. Tianhang L, Guoen F, Jianwei B, Liye M. The effect of perineural invasion on overall survival in patients with gastric carcinoma. J Gastrointest Surg 2008; 12: 1263-7.

20. Selçukbiricik F, Tural D, Büyükünal E, Serdengeçti S. Perineural invasion independent prognostic factors in patients with gastric cancer undergoing curative resection. Asian Pac J Cancer Prev 2012; 13: 3149-52.

21. Jiang N, Deng JY, Liu Y, Ke B, Liu HG, Liang H. Incorporation of perineural invasion of gastric carcinoma into the 7th edition tumornode-metastasis staging system. Tumour Biol 2014; 35: 9429-36. 